Research Paper

\title{
The role of postoperative radiotherapy for completely resected pllIA-N2 non-small cell lung cancer patients with different clinicopathological features: a systemic review and meta-analysis
}

\author{
Tingting Liu ${ }^{1^{*}}$, Yanshu $\mathrm{Mu}^{2 *}$, Jun Dang ${ }^{1}{ }^{凶}$, Guang Li ${ }^{1}$ \\ 1. Department of Radiation Oncology, The First Hospital of China Medical University, Shenyang, China \\ 2. Department of Radiation Oncology, Anshan Cancer Hospital, Anshan, China \\ * These authors contributed equally to this work. \\ $\square$ Corresponding author: Jun Dang. Department of Radiation Oncology, The First Hospital of China Medical University, 155 Nanjing Road, Heping District, \\ Shenyang, 110001, China. Tel: +86-24-83282137; Email: dangjunsy@163.com. \\ (c) Ivyspring International Publisher. This is an open access article distributed under the terms of the Creative Commons Attribution (CC BY-NC) license \\ (https://creativecommons.org/licenses/by-nc/4.0/). See http://ivyspring.com/terms for full terms and conditions.
}

Received: 2018.07.22; Accepted: 2019.05.05; Published: 2019.06.24

\begin{abstract}
Background: The role of postoperative radiotherapy (PORT) in completely resected pathological stage IIIA-N2 (pIIIA-N2) non-small cell lung cancer (NSCLC) remains controversial. This meta-analysis aimed to assess the effect of PORT in patients with pllIA-N2 NSCLC on the basis of clinicopathological features.

Methods: The PubMed, PubMed Central (PMC), Embase, Web of Science, and Cochrane Library were searched for relevant studies. The main outcomes were overall survival (OS) and disease-free survival (DFS), which were compared using the hazard ratio (HR).

Results: One randomized trial and 12 retrospective studies were eligible for the analysis. PORT significantly improved both OS [HR $=0.85 ; 95 \%$ confidence interval $(\mathrm{Cl}): 0.79-0.92]$ and DFS (HR = $0.57 ; 95 \% \mathrm{Cl}: 0.38-0.85)$ compared with non-PORT treatment in patients with multiple N2 metastases or multiple N2 station involvement. No significant difference in either OS (HR $=1.03$; 95\% Cl: $0.86-1.24)$ or DFS (HR $=1.08 ; 95 \% \mathrm{Cl}: 0.70-1.65)$ was found between PORT and non-PORT groups for patients with single N2 station involvement. No significant heterogeneity was observed. No significant differences in OS were observed between PORT and non-PORT groups for patients of different ages, sex, tumor sizes or PT stages, and histological types.

Conclusions: The findings of this meta-analysis supported a role for PORT in patients with completely resected plllA-N2 NSCLC having multiple N2 metastases and favored withholding PORT to patients with single N2 station involvement. Further prospective randomized controlled trials are needed to confirm the findings.
\end{abstract}

Key words: Non-small cell lung cancer; Stage IIIA-N2; Postoperative radiotherapy; Clinicopathological features; Meta-analysis.

\section{Introduction}

Non-small cell lung cancer (NSCLC) remains the leading cause of cancer-associated mortality globally [1-3]. Surgical resection followed by adjuvant platinum-based chemotherapy is still considered as the mainstay of treatment for most resectable cases.
However, for pathological stage IIIA-N2 (pIIIA-N2) NSCLC, the risk of locoregional recurrence (LRR) remains as high as $20 \%-40 \%$, which is independently associated with worse OS [4-6]. Based on these considerations, postoperative radiotherapy (PORT) 
has been attempted to reduce LRR and increase OS for patients with pIIIA-N2 disease. Unfortunately, a previous meta-analysis of randomized controlled trials (RCTs) demonstrated no benefit with PORT; the use of PORT could even result in a decrease in OS [7]. The meta-analysis was criticized due to the use of older two-dimensional radiation techniques, which might have led to additional toxicities no longer seen in a modern treatment population [8-9]. Recently, a number of high-quality cohort studies have demonstrated the role of PORT in patients with pIIIA-N2 NSCLC under the modern treatment model [10-12]. However, improvement in survival has been limited, and not all patients benefit from PORT in the subgroup analyses.

Actually, patients with stage pIIIA-N2 NSCLC are a heterogeneous group with different clinicopathological features. Several clinicopathological factors have been reported to be associated with different survival rates, such as number of $\mathrm{N} 2$ metastases, number of $\mathrm{N} 2$ stations involved, tumor size, histological type, age, and sex [11,13-17]. These indicate that treatments for pIIIA-N2 NSCLC should be individualized. Some studies have investigated the role of PORT according to clinicopathological features, but individual reports have been conflicting [14-26]. It is necessary to define patients who would benefit most from PORT.

In light of these critical issues, this systematic review and meta-analysis of currently available findings was performed to assess the effect of PORT in patients with pIIIA-N2 NSCLC having clinicopathological features.

\section{Materials and Methods}

This meta-analysis was conducted in accordance with the Preferred Reporting Items for Systematic Reviews and Meta-analysis (PRISMA) criteria [27].

\section{Literature search strategy}

PubMed, PubMed Central (PMC), Embase, Web of Science, and the Cochrane Library were searched for the available studies published before November 8, 2018, using the strategy as follows: (nonsmall cell lung cancer [Title/Abstract]) AND (stage IIIA-N2 [Title/Abstract]) AND ((postoperative [Title/Abstract]) OR (adjuvant [Title/Abstract])) AND ((radiotherapy [Title/Abstract]) OR (radiation therapy [Title/Abstract]) OR (chemoradiotherapy [Title/Abstract])). Further details of the search strategy are shown in Supplementary material. All published papers with available full texts were retrieved. The reference lists of retrieved studies were manually scanned for relevant additional studies missed by the electronic search. The study did not involve any experiment on humans or animals. Therefore, ethical approval was not necessary.

\section{Inclusion and exclusion criteria}

Studies were included if they met the following criteria: (1) types of studies: RCT, or prospective or retrospective cohort study; (2) types of participants: completely resected pIIIA-N2 NSCLC; (3) types of interventions: compared surgical resection with or without PORT according to clinicopathological features; and (4) outcome: reported survival (OS and/or DFS) data. If multiple articles covered the same study population, the study with the most recent and complete survival data was used. Studies were excluded if any of the following criteria were applied: (1) letters, editorials, case reports, and reviews; and (2) survival data could not be extracted from the literature.

\section{Data extraction}

The data were extracted by two investigators independently, and the consensus was reached in the case of any discrepancy for all the data. The following data were extracted from each study: first author, years of publication, duration of the study, country of origin, numbers of patients with different clinicopathological features, study design, and time-to-event data (OS and DFS). In case the studies did not report sufficient data, the authors of those studies were contacted for further information by e-mail if possible.

\section{Quality assessment}

The Newcastle-Ottawa Quality Assessment Scale (NOS) was used to assess the quality of retrospective studies [28]. The NOS comprised three items: patient selection, comparability of the study groups, and assessment of outcomes. The quality of each cohort study was scored on a scale ranging from 0 to 9 by two independent researchers. Six stars or greater was considered to denote sufficiently high-quality studies.

The methodological quality of RCT was assessed by Cochrane risk of bias tool [29], which consists of the following five domains: sequence generation, allocation concealment, blinding, incomplete data, and selective reporting. A RCT was finally rated as "low risk of bias" (all key domains indicated as low risk), "high risk of bias" (one or more key domains indicated as high risk), and "unclear risk of bias".

\section{Statistical analysis}

Data were statistically analyzed using the software Review Manager 5.3 (Cochrane Collaboration, Oxford, UK) and Stata MP14.0 (Stata Corporation, TX, USA). Hazard ratios (HRs) and their 
95\% confidence intervals (CIs) were used as summary statistics for OS in the present meta-analysis because the median survival or survival rates at a specific point in time were not expected to be reliable surrogate measures for the pooled survival analysis. Crude HRs with 95\% CIs were either extracted directly from the original reports or calculated using the Kaplan-Meier curves based on the methods of Parmer et al. [30] and Tierney et al. [31]. A statistical test for heterogeneity was performed using the chi-square $\left(\chi^{2}\right)$ and $I$-square $\left(I^{2}\right)$ tests with the significance set at $P<0.10$ and/or $I^{2}>50 \%$. If significant heterogeneity existed, a random-effects analysis model was used; otherwise, a fixed-effects model was used. The stability of the pooled results was evaluated by a sensitivity analysis in which the data of an individual study were removed each time. The funnel plot, the Begg's test [32], and the Egger's linear regression test [33] were performed to investigate any potential publication bias. A $P$ value $<0.05$ was considered to be statistically significant.

\section{Results}

\section{Literature search results and characteristics of included studies}

The literature search and study selection procedures are shown in Figure 1. The initial search from the electronic database retrieved 1417 studies. After removing the duplicates, 923 citations were identified. Of these, 827 were excluded through an abstract review. The remaining 96 studies were screened through a full-text review for further eligibility. Further, 84 studies were excluded because they did not meet the inclusion criteria. Finally, one $\mathrm{RCT}$ and 12 retrospective studies were included in the meta-analysis. Because two Surveillance, Epidemiology, and End Results (SEER)-based studies $[16,17]$ covered the same population of tumor size $>3$ $\mathrm{cm}$, the one with fewer data [16] was excluded from the subgroup analysis of tumor size $>3 \mathrm{~cm}$, and the study by Wang et al. [17] with more data was retained. The characteristics of the eligible studies are summarized in Table 1.

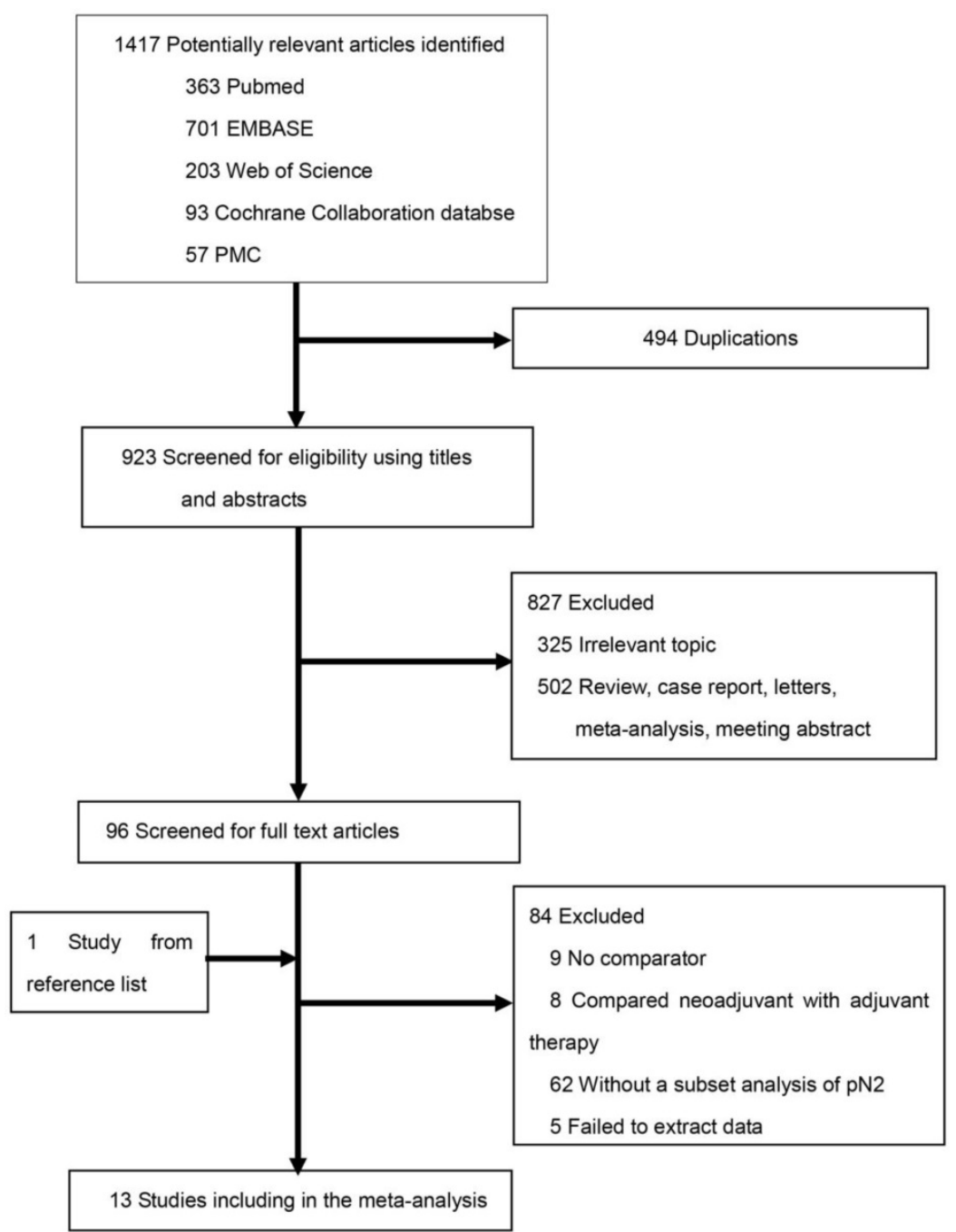

Figure 1. Literature search and selection. 


\section{Assessment of included studies}

The two researchers showed good consistency in assessing the quality of the included studies (Table 1). All of the retrospective studies demonstrated a score of $\geq 6$ (Table 2). The quality of the included RCT was relatively high (Adequate sequence generation: yes; Allocation concealment: yes; Blinding: high; Incomplete outcome: unclear; Free of selective reports: yes).

\section{Comparison of survival between the PORT and non-PORT groups for patients with different statuses of mediastinal lymph node metastasis}

PORT was associated with significantly improved OS (11 studies with 2687 patients; HR = 0.85; 95\% CI: 0.79-0.92) and DFS (4 studies with 206 patients; $\mathrm{HR}=0.57 ; 95 \% \mathrm{CI}: 0.38-0.85$ ) in patients with multiple N2 metastases or multiple N2 station involvement (Fig. 2A and 2B), and with no significant heterogeneity $\left(I^{2}=34 \% ; P=0.13\right.$; and $I^{2}=23 \%$; $P=$ 0.27 ). In the subgroup analysis, no significant difference in OS was observed between the PORT and non-PORT groups for patients with multiple N2 station involvement ( 5 studies with 402 patients; $\mathrm{HR}=$ 0.90; 95\% CI: 0.52-1.59; $P_{\text {heterogeneity }}=0.09$ ). However, when the study [20] investigating postoperative concurrent radiochemotherapy (POCRT) was removed from the subgroup analysis, the difference in OS became significant (HR $=0.72 ; 95 \% \mathrm{CI}$ : 0.51-0.99) with no significant heterogeneity $\left(I^{2}=0 \% ; P=0.46\right)$. No significant difference in either OS (8 studies with 1077 patients; $\mathrm{HR}=1.03$; 95\% CI: 0.86-1.24) or DFS (4 studies with 218 patients; $\mathrm{HR}=1.08 ; 95 \% \mathrm{CI}$ : 0.70-1.65) was found between PORT and non-PORT groups for patients with single $\mathrm{N} 2$ station involvement (Fig. $3 \mathrm{~A}$ and 3B). No significant heterogeneity was observed $\left(I^{2}=0 \% ; P=0.85\right.$; and $I^{2}=$ $0 \%, P=0.67)$.

Table 1. Baseline characteristics of included studies

\begin{tabular}{|c|c|c|c|c|c|c|c|c|c|c|}
\hline First author/ & Country & Time & No. of patients & Study & Study & POCT & RT & RT dose & Type of & NOS \\
\hline Year & of origin & range & (PORT/non-PORT) & language & design & (PORT/non-PORT) & techniques & median(Gy) & surgery & score \\
\hline Matsuguma/2008 [14] & Japan & $1986-2003$ & $45 / 46$ & English & RS & $26.7 \% / 13 \%$ & NR & 50.4 & Lob/Pne & 6 \\
\hline Wei/2017 [15] & USA & 2004-2013 & $1244 / 2090$ & English & RS & NA & NR & NR & Sub/Lob/Pne & 7 \\
\hline Kou/2018 [16] & USA & $2004-2013$ & $1106 / 1843$ & English & RS & NA & NR & NR & NR & 7 \\
\hline Wang/2017 [17] & USA & 2004-2013 & $1198 / 2179$ & English & RS & NA & NR & NR & Lob/Pne & 7 \\
\hline $\mathrm{Du} / 2009$ [18] & China & $2000-2005$ & $104 / 255$ & Chinese & RS & $73.1 \% / 51.4 \%$ & $2 \mathrm{D}$ & NR & Lob/Pne & 6 \\
\hline $\mathrm{Xu} / 2018$ [19] & China & 2009-2012 & $89 / 157$ & English & RS & $98.9 \% / 57.3 \%$ & $3 \mathrm{D}$ & 50.4 & Lob/Pne & 7 \\
\hline Sun/2017 [20] & Korea & 2009-2014 & $51 / 50$ & English & $\mathrm{RCT}$ & $100 \% * / 100 \%$ & $3 \mathrm{D}$ & 50 & Lob/Bilo/Pne & - \\
\hline Kim/2014 [21] & Korea & $2000-2011$ & $41 / 178$ & English & RS & $100 \% / \mathrm{NA}$ & $2 \mathrm{D}+3 \mathrm{D}$ & 54 & Lob/Bilo/Pne & 7 \\
\hline Hui/2014 [22] & China & $2003-2005$ & $96 / 125$ & English & RS & NA & $2 \mathrm{D}+3 \mathrm{D}$ & 60 & Lob/Pne & 7 \\
\hline $\mathrm{Cao} / 2014$ [23] & China & $2008-2009$ & $39 / 179$ & English & RS & $100 \% / 71.5 \%$ & $3 \mathrm{D}$ & 50.4 & Lob/Bilo/Pne/Wed & 7 \\
\hline Pang/2017 [24] & USA & $2004-2011$ & $9040 / 5419$ & English & RS & NA & NR & NR & Lob/Bilo/Pne/Wed & 7 \\
\hline Sawyer/1997 [25] & USA & 1987-1993 & $88 / 136$ & English & RS & NA & NR & 50.4 & Lob/Pne/Wed & 6 \\
\hline Chen/2009 [26] & China & 1987-2004 & $46 / 46$ & Chinese & RS & NA & NR & 56 & Lob/Pne & 6 \\
\hline
\end{tabular}

Abbreviations: PORT, postoperative radiotherapy; POCT, postoperative chemotherapy; RT, radiotherapy; NOS, Newcastle-Ottawa Quality Assessment Scale score; NR, not reported; 2D, two-dimensional radiotherapy; 3D, three-dimensional conformed radiotherapy; Lob, lobectomy; Pne, pneumonectomy; Sub, sublobectomy; Bilo, Bilobectomy; Wed, wedge resection; RCT, randomized controlled trial; RS, retrospective cohort study.

*, concurrent chemoradiotherapy.

Table 2. Quality assessment of twelve retrospective studies using the Newcastle-Ottawa scale.

\begin{tabular}{|c|c|c|c|c|c|c|c|c|c|c|}
\hline \multirow{2}{*}{$\begin{array}{l}\text { First author/ } \\
\text { year }\end{array}$} & \multicolumn{4}{|c|}{ Selection } & \multicolumn{2}{|c|}{ Comparablity } & \multicolumn{3}{|c|}{ Outcome } & \multirow[t]{2}{*}{ Score } \\
\hline & Item1 & Item2 & Item3 & Item4 & Item5 & Item6 & Item7 & Item8 & Item9 & \\
\hline Matsuguma/2008[14] & ※ & $※$ & $※$ & $※$ & $※$ & - & - & $※$ & - & 6 \\
\hline Wei/2017[15] & $※$ & $※$ & $※$ & $※$ & $※$ & - & $※$ & $※$ & - & 7 \\
\hline Kou/2018[16] & $※$ & $※$ & $※$ & $※$ & $※$ & - & $※$ & $※$ & - & 7 \\
\hline Wang/2017[17] & $※$ & $※$ & $※$ & $※$ & $※$ & - & $※$ & $※$ & - & 7 \\
\hline $\mathrm{Du} / 2009[18]$ & $※$ & $※$ & $※$ & $※$ & $※$ & - & - & $※$ & - & 6 \\
\hline $\mathrm{Xu} / 2018[19]$ & $※$ & $※$ & $※$ & $※$ & $※$ & - & $※$ & $※$ & - & 7 \\
\hline $\mathrm{Kim} / 2014[21]$ & $※$ & $※$ & $※$ & $※$ & $※$ & - & $※$ & $※$ & - & 7 \\
\hline Hui/2014[22] & $※$ & $※$ & $※$ & $※$ & $※$ & - & $※$ & $※$ & - & 7 \\
\hline Cao/2014[23] & $※$ & $※$ & $※$ & $※$ & $※$ & - & $※$ & $※$ & - & 7 \\
\hline Pang/2017[24] & $※$ & $※$ & $※$ & $※$ & $※$ & - & $※$ & $※$ & - & 7 \\
\hline Sawyer/1997[25] & $※$ & $※$ & $※$ & $※$ & $※$ & - & - & $※$ & - & 6 \\
\hline Chen/2009[26] & - & - & $※$ & $※$ & $※$ & - & $※$ & $※$ & $※$ & 6 \\
\hline
\end{tabular}

Abbreviations: -, zero point; $※$, one point. Item 1: representativeness of the exposed cohort; Item 2: selection of the nonexposed cohort; Item 3 : ascertainment of exposure; Item 4: demonstrating that the outcome of interest was not present at the start of the study; Item 5: comparability of cohorts on the basis of the design (study controls for the most important factor); Item 6: comparability of cohorts on the basis of the design (study controls for other additional factors); Item 7: assessment of outcome; Item 8:

follow-up long enough for outcomes to occur; and Item 9: adequacy of follow-up of cohorts. 


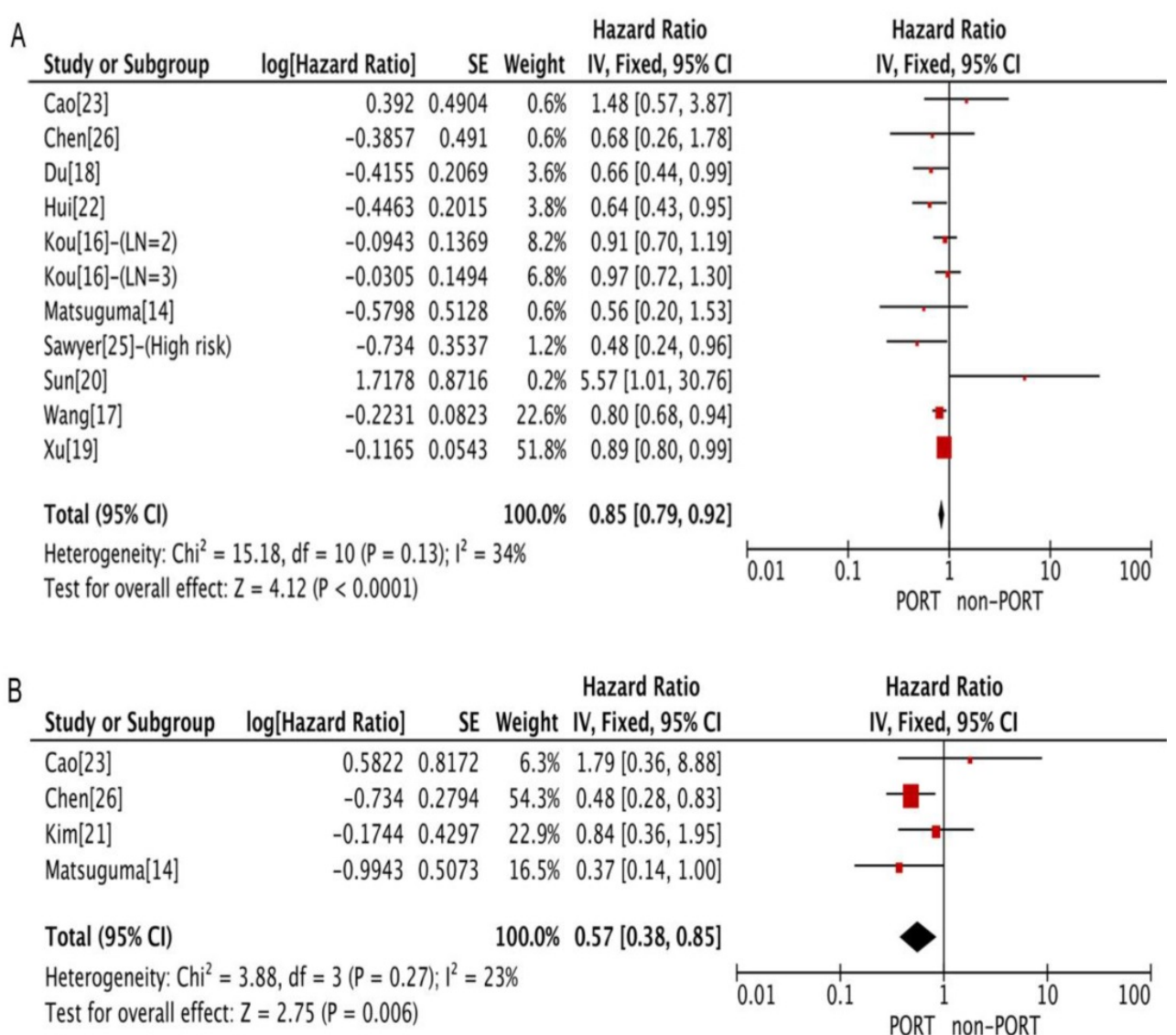

Figure 2. Forest plots of HRs for OS and DFS in patients with multiple N2 metastases or multiple N2 station involvement. (A) OS; (B) DFS. Cl, Confidence interval; DFS, disease-free survival; IV, inverse variance method; OS, overall survival; PORT, postoperative radiotherapy; SE, standard error.

A

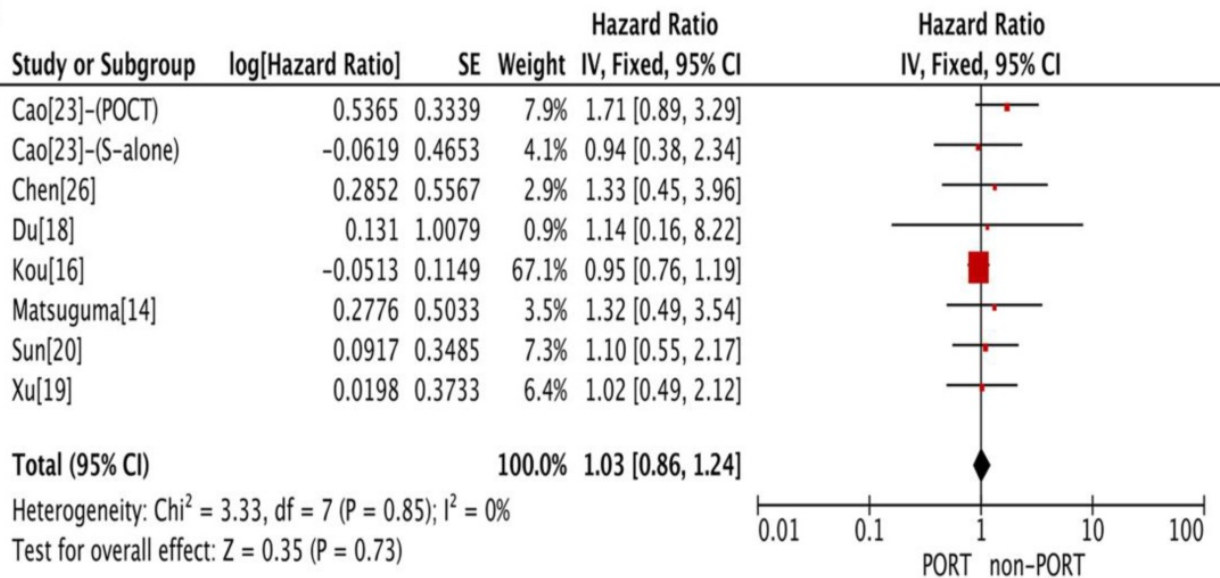

B

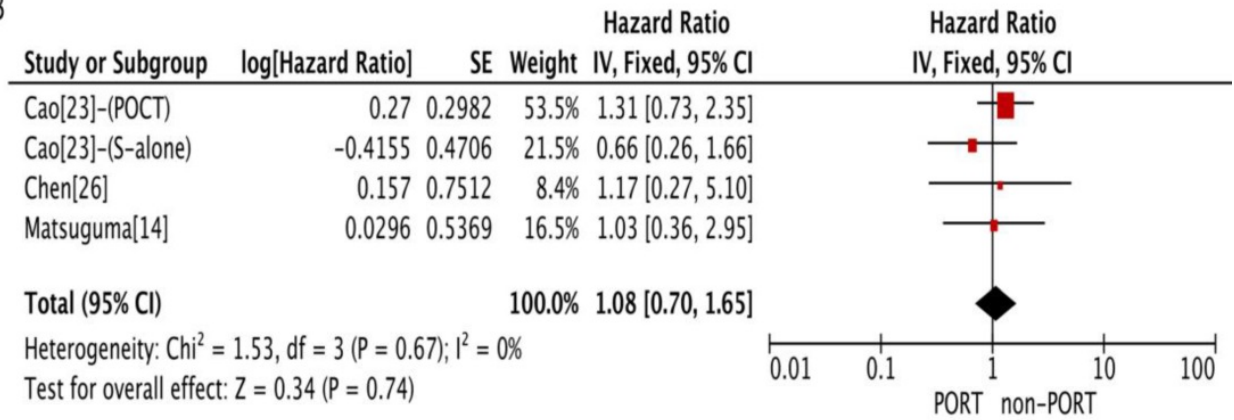

Figure 3. Forest plots of HRs for OS and DFS in patients with single N2 station involvement. (A) OS; (B) DFS. PORT: postoperative radiotherapy; Cl, Confidence interval; DFS, disease-free survival; IV, inverse variance method; OS, overall survival; PORT, postoperative radiotherapy; SE, standard error. 
A

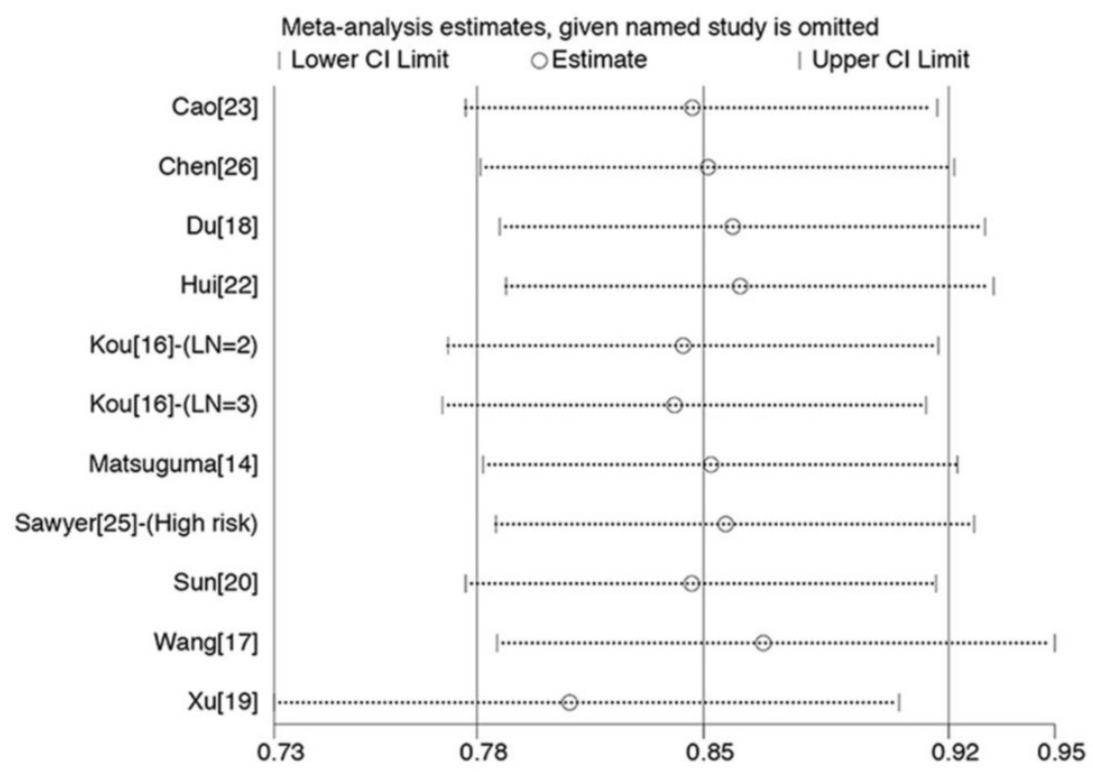

B

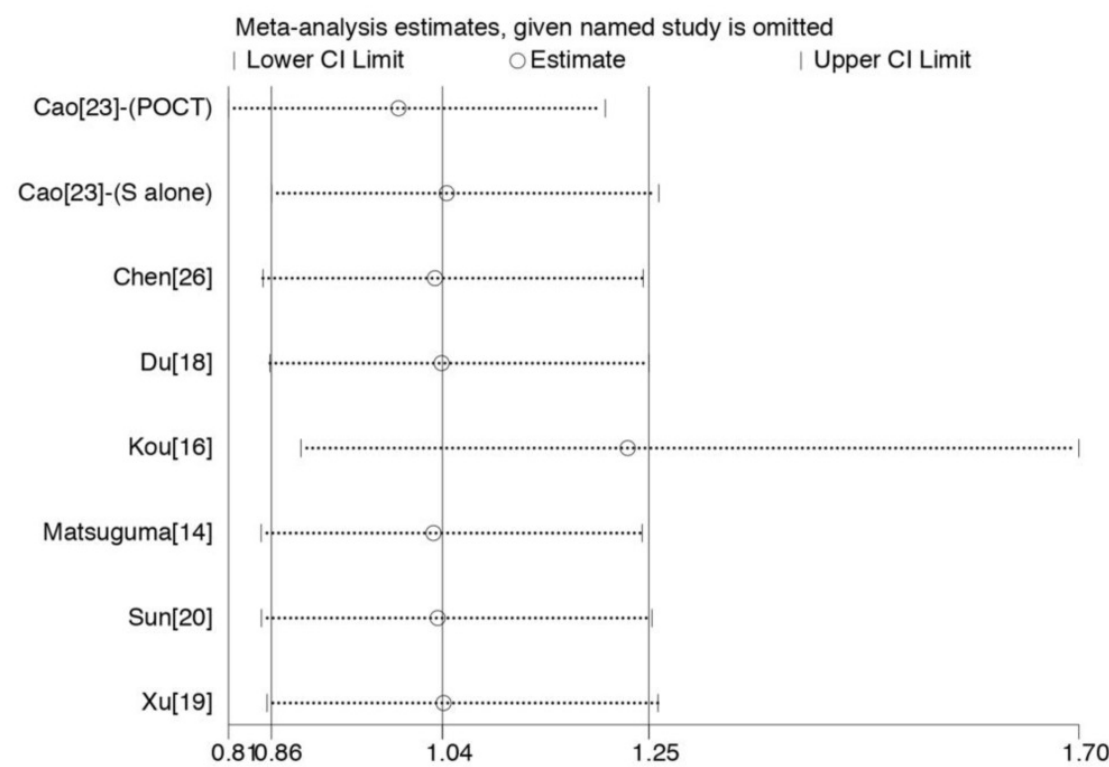

Figure 4. Sensitivity analysis for comparing OS between the PORT and non-PORT groups. (A) In studies investigating multiple N2 metastases or multiple N2 station involvement; (B) in studies investigating single N2 station involvement. PORT, Postoperative radiotherapy.

Sensitivity analyses were carried out to assess whether individual studies influenced the results. When individual studies were removed one at a time from the analyses for OS, the corresponding pooled HRs were not markedly altered by any single study (Fig. $4 \mathrm{~A}$ and $4 \mathrm{~B}$ ), confirming the stability of the presented results.

The Begg's and Egger's test results indicated no publication bias in terms of OS in studies investigating PORT for multiple N2 metastases or multiple N2 station involvement $(P=1.0$ for Begg's test; $P=0.82$ for Egger's test) and studies investigating PORT for single N2 station involvement $(P=0.90$ for Begg's test; $P=0.15$ for Egger's test).

\section{Comparison of OS between the PORT and non-PORT groups in patients with other clinicopathological features}

No significant difference in OS was observed between the PORT and non-PORT groups for either patients with tumor size $>3 \mathrm{~cm}$ or T2-3 tumors $(8$ studies with 8311 patients; $\mathrm{HR}=1.03 ; 95 \% \mathrm{CI}$ : $0.87-1.22$ ) (Fig. 5A) or patients with tumor size $\leqq 3 \mathrm{~cm}$ or T1 tumors (4 studies with 6742 patients; $\mathrm{HR}=1.09$; 95\% CI: 0.80-1.49) (Fig. 5B) and with significant heterogeneity $\left(I^{2}=83 \% ; P<0.001\right.$; and $I^{2}=86 \%$; $P<$ 0.001).

No significant differences in OS were observed between the PORT and non-PORT groups for age $\geq 60$ 


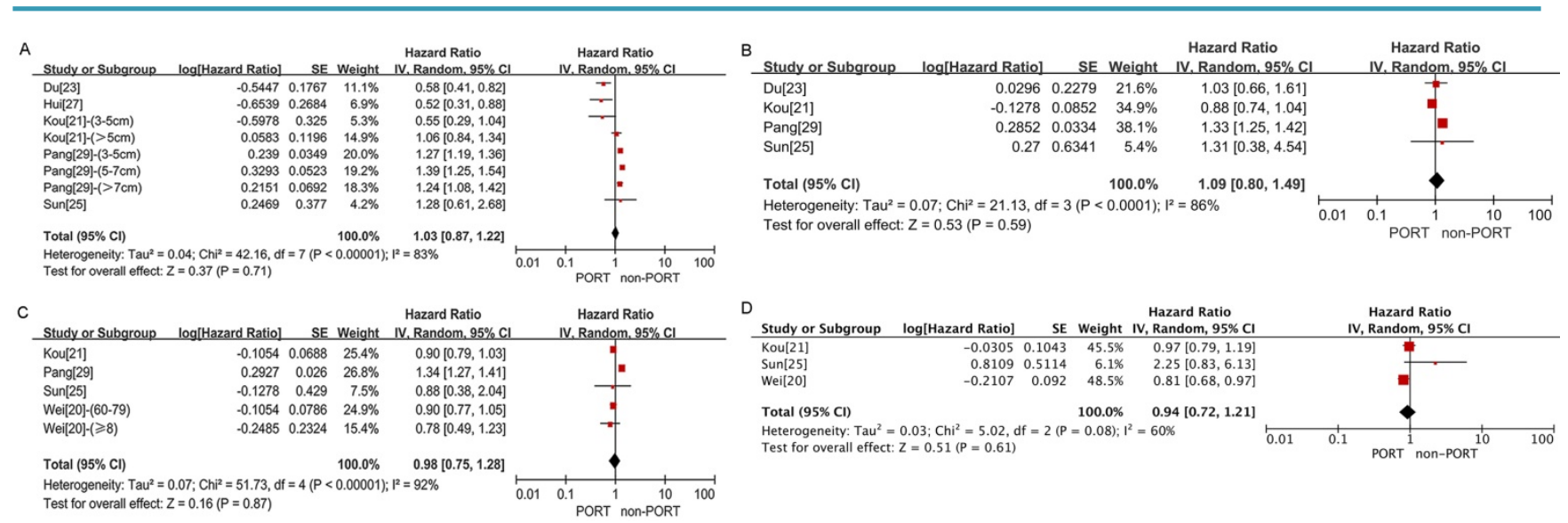

Figure 5. Forest plots of HRs for OS in patients with other clinicopathological features. (A) Tumor size $>3 \mathrm{~cm}$ or T2-3 tumors; (B) tumor size $\leqq 3 \mathrm{~cm}$ or T1 tumors; (C) age $\geq 60$ years; (D) age $<60$ years.

years (5 studies with 10,044 patients; $\mathrm{HR}=0.98 ; 95 \%$ CI: 0.75-1.28; $P_{\text {heterogeneity }}<0.001$ ) (Fig. 5C), age $<60$ years ( 3 studies with 1202 patients; $\mathrm{HR}=0.94 ; 95 \% \mathrm{CI}$ : 0.72-1.21; $P_{\text {heterogeneity }}=0.08$ ) (Fig. 5D), patients with squamous cell carcinoma (4 studies with 3814 patients; HR $=0.92 ; 95 \%$ CI: $0.64-1.33 ; P_{\text {heterogeneity }}<$ 0.001) (Figure. 6A), patients with adenocarcinoma (3 studies with 8129 patients; $\mathrm{HR}=1.02$; $95 \% \mathrm{CI}$ : 0.52-2; $\left.P_{\text {heterogeneity }}=0.05\right)($ Fig. 6B), male (3 studies with 8746 patients; HR $=0.99 ; 95 \%$ CI: $0.65-1.5, P_{\text {heterogeneity }}<$ 0.001) (Fig. 6C); and female (3 studies with 8014 patients; $\mathrm{HR}=1.28 ; 95 \% \mathrm{CI}: 0.99-1.65 ; P_{\text {heterogeneity }}=$ 0.005) (Fig. 6D).

\section{Discussion}

To our knowledge, this is the first meta-analysis to evaluate the role of PORT in completely resected pIIIA-N2 NSCLC on the basis of clinicopathological features. It showed that PORT significantly improved OS and DFS compared with non-PORT treatment in patients with multiple N2 metastases or multiple N2 station involvement. No significant difference in either OS or DFS was observed between the PORT and non-PORT groups for patients with single N2 station involvement. No significant differences in OS were found between the PORT and non-PORT groups for patients of different ages, sex, tumor sizes or $\mathrm{pT}$ stages, and histological types, but heterogeneity was evident across the studies.

Multiple N2 metastases or multiple N2 station involvement is associated with a high local recurrence rate compared with single N2 station involvement [14, 34]. Therefore, a number of studies investigated the efficacy of PORT for pIIIA-N2 NSCLC according to the N2 metastasis status. Several retrospective studies
$[14,18,19]$, including two population-based studies $[16,17]$, demonstrated the benefit of PORT in terms of improving OS for patients with multiple N2 metastases or multiple N2 station involvement, but not for patients with single N2 station involvement. However, the subgroup analysis of a recent randomized phase II study [20] revealed that postoperative chemotherapy (POCT) alone was favored compared with POCRT in patients with multiple N2 station involvement. In the present meta-analysis, PORT improved OS and DFS for patients with multiple N2 LN metastases or multiple $\mathrm{N} 2$ station involvement, but not for patients with single N2 station involvement. However, no significant difference in OS was found for multiple N2 station involvement in the subgroup analysis. However, when the study [20] using POCRT was removed from the subgroup analysis, the difference in OS became significant, with no significant heterogeneity. A more recent National Cancer Database (NCDB)-based study [35] showed that postoperative sequential chemoradiotherapy was associated with improved survival over POCRT for completely resected pN2 NSCLC; toxicity-related factors might help explain these results. Other schedules of PORT, such as early or late treatment period of PORT [14] and PORT with or without POCT [21], were also reported as related to different survival outcomes. The results suggested that the schedule of PORT might affect the efficacy of PORT. However, a subgroup analysis according to the schedule of PORT could not be performed due to the lack of detailed information in individual studies. Further well-designed, prospective studies are needed to identify the optimal schedule of PORT. 

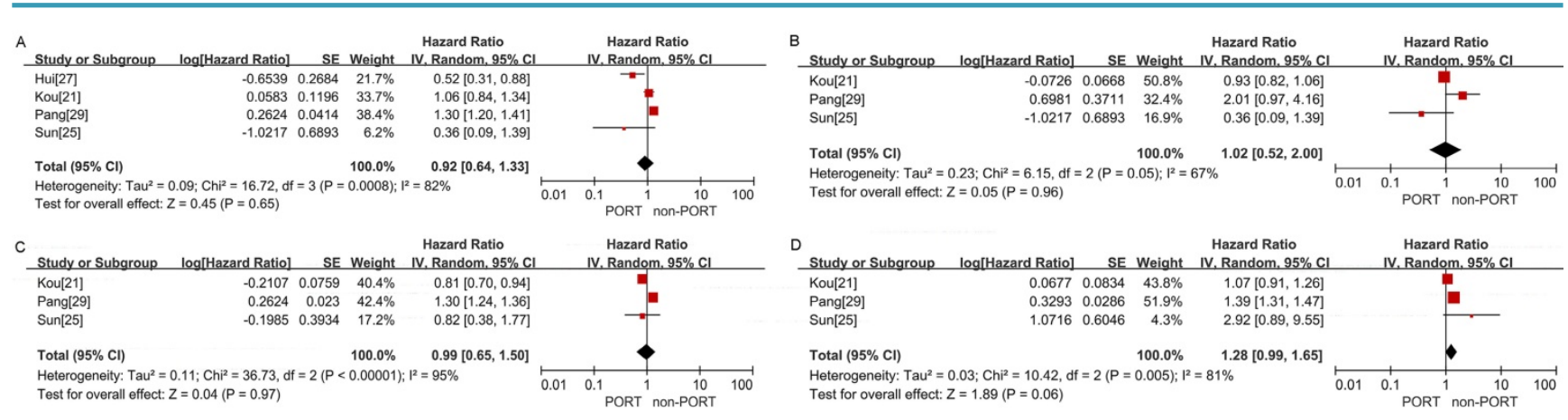

Figure 6. Forest plots of HRs for OS in patients with other clinicopathological features. (A) Squamous cell carcinoma; (B) adenocarcinoma; (C) male; (D) female.

The prognostic value of tumor size and $\mathrm{pT}$ stage in pIIIA-N2 NSCLC has also been investigated in some studies [36-38]; the results indicated that tumor size and $\mathrm{pT}$ stage might be prognostic indicators for survival. A few studies tried to assess the efficacy of PORT according to the $\mathrm{pT}$ stage or tumor size, but yielded conflicting results $[12,15,16,22]$. The present meta-analysis showed no significant differences in OS between the PORT and non-PORT groups for either patients with tumor $>3.0 \mathrm{~cm}$ or pT2-3 tumor or those with tumor $3.0 \mathrm{~cm}$ or less or pT1 tumor. However, the heterogeneity was evident across the studies. One possible source of heterogeneity was the various cut-off points of tumor size used in individual studies when assessing the efficacy of PORT according to tumor size. Thus, the present findings were not solid. Hence, the role of PORT for patients with different tumor sizes or $\mathrm{pT}$ stages needs further investigation.

The present meta-analysis also showed no significant differences in OS between the PORT and non-PORT groups for patients of different ages, sex, and histological types, which were also reported to be potential pathological factors. However, no conclusion could be drawn due to less number of included studies or small sample size and significant heterogeneities.

The present meta-analysis had several limitations. First, almost all the available data were extracted from retrospective studies. These observational data had the inherent limitations, such as potential imbalance in clinical factors. However, according to the Crochrane Handbook version 5.1.0, reviews of non-randomized studies are likely to be undertaken when the question of interest cannot be answered by a review of randomized trials. Currently, there is little trial on the effect of PORT in patients with pIIIA-N2 NSCLC on the basis of clinicopathological features. In our meta-analysis, all of the included studies had a NOS $\geq 6$. No significant heterogeneity was observed for patients with multiple $\mathrm{N} 2$ metastases or multiple N2 station or single N2 station involvement. The sensitivity analysis for OS revealed that the corresponding pooled HRs were not markedly altered by any single study, confirming the stability of the presented results. Of cause, compared with RCTs, the evidence of retrospective studies is lower, but the findings of our meta-analysis seem to be useful to inform the design of a subsequent randomized trial. Second, a few HRs were not directly reported in the texts and hence calculated from the Kaplan-Meier curves, resulting in bias and error. Finally, the number of included studies in several subgroups, such as age, sex, and histological type, was less with significant heterogeneity. Therefore, the conclusion based on these factors should be discreet.

\section{Conclusions}

Evidences from the present meta-analysis supported a role for PORT in patients with completely resected pIIIA-N2 NSCLC having multiple N2 metastases and favored withholding PORT to patients with single N2 station involvement. Further prospective RCTs are needed to confirm the findings.

\section{Supplementary Material}

Search strategy.

http://www.jcancer.org/v10p3941s1.pdf

\section{Competing Interests}

The authors have declared that no competing interest exists.

\section{References}

1. Ettinger DS, Wood DE, Aisner DL, et al. Non-Small Cell Lung Cancer, Version 5.2017, NCCN Clinical Practice Guidelines in Oncology. J Natl Compr Canc Netw. 2017;15:504-35.

2. De Marinis F, Ciardiello F, Baas P, et al. 30 Immunotherapy in advanced NSCLC-from the 'tsunami' of therapeutic knowledge to a clinical practice algorithm: results from an international expert panel meeting of the Italian Association of Thoracic Oncology (AIOT). ESMO Open. 2018;3:e000298.

3. Goeckenjan G, Sitter H, Thomas M, et al. Prevention, diagnosis, therapy, and follow-up of lung cancer: Interdisciplinary guideline of the German Respiratory Society and the German Cancer Society. Pneumologie. 2011;65:e51-75.

4. Arriagada R, Bergman B, Dunant A, et al. Cisplatin-based adjuvant chemotherapy in patients with completely resected non-small-cell lung cancer. N Engl J Med. 2004;350:351-60.

5. Winton T, Livingston R, Johnson D, et al. Vinorelbine plus cisplatin vs. observation in resected non-small-cell lung cancer. N Engl J Med. 2005;352: 2589-97.

6. Douillard JY, Rosell R, De Lena M, et al. Adjuvant vinorelbine plus cisplatin versus observation in patients with completely resected stage IB-IIIA 
non-small-cell lung cancer (Adjuvant Navelbine International Trialist Association [ANITA]): a randomised controlled trial. Lancet Oncol. 2006;7:719-27.

7. Postoperative radiotherapy in non-small-cell lung cancer: systematic review and meta-analysis of individual patient data from nine randomised controlled trials. PORT Meta-analysis Trialists Group. Lancet. 1998; 352:257-63.

8. Munro AJ. What now for postoperative radiotherapy for lung cancer? Lancet 1998; 352:250-51.

9. Machtay M, Lee JH, Shrager JB, et al. Risk of death from intercurrent disease is not excessively increased by modern postoperative radiotherapy for high-risk resected non-small- cell lung carcinoma. J Clin Oncol. 2001;19:3912-17.

10. Zou $\mathrm{B}, \mathrm{Xu} \mathrm{Y}, \mathrm{Li} \mathrm{T}$, et al. A multicenter retrospective analysis of survival outcome following postoperative chemoradiotherapy in non-small-cell lung cancer patients with N2 nodal disease. Int J Radiat Oncol Biol Phys. 2010;77:321-28.

11. Robinson CG, Patel AP, Bradley JD, et al. Postoperative radiotherapy for pathologic N2 non-small-cell lung cancer treated with adjuvant chemotherapy: a review of the National Cancer Data Base. J Clin Oncol. 2015;33:870-76.

12. Douillard JY, Rosell R, De Lena M, Riggi M, Hurteloup P, Mahe MA. Adjuvant Navelbine International Trialist Association. Impact of postoperative radiation therapy on survival in patients with complete resection and stage I, II, or IIIA non-small-cell lung cancer treated with adjuvant chemotherapy: the adjuvant Navelbine International Trialist Association (ANITA) Randomized Trial. Int J Radiat Oncol Biol Phys. 2008;72:695-701.

13. Mikell JL, Gillespie TW, Hall WA, et al. Postoperative radiotherapy is associated with better survival in non-small cell lung cancer with involved N2 lymph nodes: results of an analysis of the National Cancer Data Base. J Thorac Oncol. 2015;10:462-71.

14. Matsuguma H, Nakahara R, Ishikawa $Y$, et al. Postoperative radiotherapy for patients with completely resected pathological stage IIIA-N2 non-small cell lung cancer: focusing on an effect of the number of mediastinal lymph node stations involved. Interact Cardiovasc Thorac Surg. 2008;7:573-77.

15. Wei S, Xie M, Tian J, Song X, Wu B, Liu L. Propensity score-matching analysis of postoperative radiotherapy for stage IIIA-N2 non-small cell lung cancer using the Surveillance, Epidemiology, and End Results database. Radiat Oncol. 2017;12:96.

16. Kou P, Wang $\mathrm{H}$, Lin J, Zhang $\mathrm{Y}$, Yu J. Male patients with resected IIIA-N2 non-small-cell lung cancer may benefit from postoperative radiotherapy: a population-based survival analysis. Future Oncol. 2018 May 29.

17. Wang S, Ma Z, Yang X, et al. Choice of postoperative radiation for stage IIIA pathologic N2 non-small cell lung cancer: impact of metastatic lymph node number. Radiat Oncol. 2017;12:207.

18. Du F, Yuan Z, Wang J, et al. The Role of Postoperative Radiotherapy on Stage N2 Non-small Cell Lung Cancer. Zhongguo Fei Ai Za Zhi. 2009;12:1164-68. [In Chinese]

19. Xu Y, Li J, Wang J, et al. Association between clinicopathological factors and postoperative radiotherapy in patients with completely resected pathological N2 non-small cell lung cancer. Oncol Lett. 2018;15:2641-50.

20. Sun JM, Noh JM, Oh D, et al. Randomized Phase II Trial Comparing Chemoradiotherapy with Chemotherapy for Completely Resected Unsuspected N2-Positive Non-Small Cell Lung Cancer. J Thorac Oncol. 2017; 12:1806-13.

21. Kim $\mathrm{BH}, \mathrm{Kim} \mathrm{HJ}, \mathrm{Wu} \mathrm{HG}$, et al. Role of postoperative radiotherapy after curative resection and adjuvant chemotherapy for patients with pathological stage N2 non-small-cell lung cancer: a propensity score matching analysis. Clin Lung Cancer. 2014;15:356-64

22. Hui Z, Dai H, Liang J, et al. Selection of proper candidates with resected pathological stage IIIA-N2 non-small cell lung cancer for postoperative radiotherapy. Thorac Cancer. 2015;6:346-53.

23. Cao Q, Zhang B, Zhao $\mathrm{L}$, et al. Reappraisal of the role of postoperative radiation therapy in patients with pIIIa-N2 non-small cell lung cancer: A propensity score matching analysis. Thorac Cancer. 2015;6:570-78.

24. Pang Z, Yang Y, Ding N, et al. Optimal managements of stage IIIA (N2) non-small cell lung cancer patients: a population-based survival analysis. J Thorac Dis. 2017:9:4046-56.

25. Sawyer TE, Bonner JA, Gould PM, et al. Effectiveness of postoperative irradiation in stage IIIA non-small cell lung cancer according to regression tree analyses of recurrence risks. Ann Thorac Surg. 1997;64:1402-8.

26. Chen J, Zhang X, Zeng Y, et al. An analysis of postoperative radiotherapy for patients with completely resected stage IIIa(N2) non-small cell lung cancer. Journal of Chongqing Medical University. 2009;34:1566-70. [In Chinese]

27. Moher D, Liberati A, Tetzlaff J, Altman DG; PRISMA Group. Preferred reporting items for systematic reviews and meta-analyses: the PRISMA statement. Int J Surg. 2010;8:336-41.

28. Stang A. Critical evaluation of the Newcastle-Ottawa scale for the assessment of the quality of nonrandomized studies in meta-analyses. European journal of epidemiology. 2010;25:603-5.

29. Higgins JP, Altman DG, Gotzsche PC, et al. The Cochrane Collaboration's tool for assessing risk of bias in randomised trials. BMJ. 2011;343:d5928.

30. Parmar MK, Torri V, Stewart L. Extracting summary statistics to perform meta-analyses of the published literature for survival endpoints. Stat Med. 1998;17:2815-34.
31. Tierney JF, Stewart LA, Ghersi D, Burdett S, Sydes MR. Practical methods for incorporating summary time-to-event data into meta-analysis. Trials. 2007;8:16.

32. Begg $\mathrm{CB}$, Mazumdar M. Operating characteristics of a rank correlation test for publication bias. Biometrics. 1994;50:1088-101.

33. Egger M, Davey Smith G, Schneider M, Minder C. Bias in meta-analysis detected by a simple, graphical test. BMJ. 1997;315:629-34.

34. Ichinose Y, Kato H, Koike T, et al; Japan Clinical Oncology Group. Overall survival and local recurrence of 406 completely resected stage IIIa-N2 non-small cell lung cancer patients: questionnaire survey of the Japan Clinical Oncology Group to plan for clinical trials. Lung Cancer. 2001;34:29-36.

35. Moreno AC, Haque W, Verma V, Fang P, Lin SH. Concurrent Versus Sequential Chemoradiation Therapy in Completely Resected Pathologic N2 Non-Small Cell Lung Cancer: Propensity-Matched Analysis of the National Cancer Data Base. Ann Surg Oncol. 2018;25:1245-53.

36. Sura K, Grills IS, Vu CC, Stevens CW, Ye H, Guerrero TM. Improved Survival With Increased Time-To-Radiation and Sequential Chemotherapy After Surgery for pN2 Non-Small-cell Lung Cancer. Clin Lung Cancer. 2018;19:e185-94.

37. Kim HK, Cho JH, Choi YS, et al. Outcomes of neoadjuvant concurrent chemoradiotherapy followed by surgery for non-small-cell lung cancer with N2 disease. Lung Cancer. 2016;96:56-62.

38. Uehara $H$, Nakao $M$, Mun $M$, et al. Significant prognostic factors for completely resected pN2 non-small cell lung cancer without neoadjuvant therapy. Ann Thorac Cardiovasc Surg. 2015;21:345-53. 